\title{
MATERIAL IDENTIFICATION OF SOFT TISSUE USING MEMBRANE INFLATION*
}

\author{
Alain Wineman, Dolglas Wilson and John W. Melvin \\ Department of Applied Mechanics and Engineering Science, \\ The University of Michigan, Ann Arbor, MI 48109, U.S.A.
}

\begin{abstract}
The constitutive equation for large elastic deformations is often used to model the mechanical response of soft tissue. This paper is concerned with the applications of the method of material identification to the determination of the strain energy density functions $(W)$ in such a mode, under the assumption that the tissue is incompressible and isotropic. It is shown that an identification experiment based on inflation by lateral pressure of an initially fiat circular membrapeous specimen has a number of advantages. These are: the method of clamping the specimen, the ease of labelling material particles and measuring current coordinates, the easily determined domain of identification of $W$, and a means of systematically determining $W$ over a large deformation range. An example in the form of a hypothetical experiment is presented.
\end{abstract}

\section{INTRODUCTION}

The constitutive equation for large elastic deformations is often used to model the mechanical response of soft biological tissues as well as rubbery materials. Its application depends on the knowledge of the strain energy density function $(W)$ for the material under consideration. Forms for $W$ for incompressible rubbery materials are reasonably well established. On the other hand, a significant amount of activity is concerned with the determination of strain energy density functions for soft tissues.

The procedures which have been used to establish forms for $W$ for rubber appear to be the guide for some current approaches for the determination of $W$ for soft tissue. It will be useful, for present purposes, to briefly review the determination of $W$ for rubber. The initial forms for $W$ were developed from an experimental program based on subjecting specimens to unequal biaxial homogeneous deformations. In order to assess the Mooney form of $W$ in predicting nonhomogeneous deformations, Adkins and Rivlin (1952) used it in the calculation of the deformed profiles of a clamped, initially flat, circular rubber membrane which had been pressurized on one side. These were then compared with actual profiles which had been previously measured by Treloar (1944). Agreement between the measured and calculated profiles was satisfactory at low inflation levels but less so at higher levels. Later, Klingbeil and Shield (1964), and then Hart-Smith and Crisp (1967) introduced improved forms for $W$ for which the match between measured and calculated profiles was very good.

Recently, a method has been suggested which combines these stages of construction from homogeneous deformations, verification against nonhomogeneous deformations and subsequent improve-

* Received 26 July 1978; in revised form Il May 1979 ment. Called material identification, it is discussed in detail by Iding et al. (1974). Material identification, as a method for determining the strain energy density function $W$, consists of four elements : (1) an experimental configuration, not necessarily producing homogeneous deformations, which is convenient for specimen support, load application and measurement of the motion of specimen particles during deformation; (2) a representation for the strain energy density function in terms of undetermined parameters; (3) a method for calculating the coordinates of material particles for any reasonable choice of these parameters from a mathematical model of the experiment ; (4) a method for adjusting these parameters in order to minimize the error between measured and computed coordinates.

Identification appears to be a very useful technique for determining the strain energy density function for soft tissues. Experiments are not limited to the imposition of homogeneous uniaxial or biaxial deformations in which the gripping of specimens and load application sometimes pose problems. Instead, in accordance with element (1) above, a specimen can be subjected to a convenient non-homogeneous deformation which avoids these problems. Such a specimen can be regarded as an assemblage of an infinite number of local homogeneous deformations. In effect, $W$ is simultaneously fitted to data from these homogeneous deformations and shown to lead to accurate predictions of the non-homogeneous deformations involved in the experiment.

Iding et al. illustrated the identification procedure with a hypothetical experiment which utilized a flat tensile specimen with monotonically decreasing width. The experiment served to point out a difficulty with the procedure. The arguments of $W$, being strain invariants, depend on the experiment and are not generally known a priori. It may be difficult to determine their domain of values from experimental data. Thus, it is necessary to determine the domain on which $W$ is defined as well as its values on that domain. 
The purpose of this paper is twofold : (i) to show that the circular membrane inflation test used in the rubber experiments is especially suitable for the identification procedure, and (ii) to point out the promise of the procedure for the determination of the material properties for many kinds of soft tissue. It will be shown that, in addition to meeting the requirements of element (1) in a simple and direct manner, this test enables the domain of definition of $W$ to be determined at each inflation level. Furthermore, by considering increasing inflation levels, $W$ can be systematically determined over as large a domain as desired.

Inflation techniques have been used to a limited extent in the determination of properties of some soft tissues. For example, the technique has been used as a basis for the measurement of material properties during equal biaxial extension, a state which exists at the center of the membrane, Melvin et al. (1975). Measurements have been made on samples of human and monkey blood vessel, aorta and diaphragm at the University of Michigan Highway Safety Research Institute. Recently, in a different approach, Miller et al. (1979), the circular sheet inflation test has been carried out using human fetal membrane. This tissue was modelled by nonlinear elasticity with a Mooney form of $W$. The constants in this model were determined from the measurement of the deformed surface of the membrane.

There are limitations to the use of the circular membrane inflation test as a basis for identification. Clearly, it can only be used to determine material properties for inplane biaxial stretching. A different test configuration would be necessary for other deformation states. Even with this limitation, the test should be appropriate to the material loading conditions for many soft tissues. This test also restricts consideration to membraneous tissue whose consistency is such that it can be clamped using a retaining ring. Many kinds of tissue samples, such as mentioned before, fall into this category.

The scope of the present work is confined to the presentation of the theory and a numerical example. Experimental identification of the elastic properties of an actual material will be presented in a later paper.

The experiment is described in Section 2. Its ma-

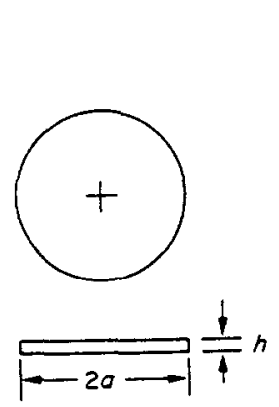

(a)
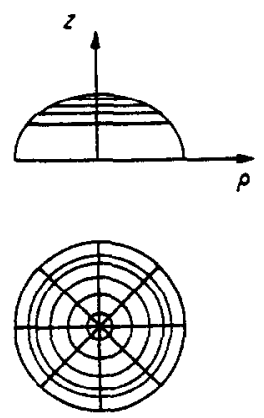

(b)

Fig. 1. Schematic version of the inflation test. (a) Undeformed specimen. (b) Inflated specimen and grid system. thematical model is presented in Section 3. For convenience, it will be assumed that the materials under consideration are incompressible and isotropic. The former assumption is reasonable. In later work it may be possible to extend the method discussed here to allow for orthotropy. The determination of the domain of definition of $W$ for each inflation level is discussed in Section 4. The details of elements (2), (3) and (4), pertaining to the inflation test, are presented in Sections 5,6 and 7, respectively. Finally, the results of a numerically simulated identification experiment are discussed in Section 8.

\section{MEMBRANE INFLATION TEST}

A brief description of the experiment is presented here. More details will be given in a later paper.

A schematic version of the test is shown in Fig. 1. The specimen is an initially plane membrane, assumed to be of uniform thickness $h_{0}$. It is clamped with an Oring seal, thereby creating the boundary of radius $a_{0}$. The membrane is inflated by controlled, continuously monitored air pressure applied to one side. This method avoids material failure at the clamping support and allows failure in the central region where the greatest distension and thickness reduction occurs.

Before deformation, the specimen is marked with a grid of concentric circular and radial lines. This can be done, for example, with an inked rubber stamp. The particles to be followed during deformation are the intersections of these lines. A mirror is mounted next to the specimen at an angle to the vertical. A movie camera mounted overhead photographs the top and side views of the specimen simultaneously during inflation. By determining the particle coordinates from a movie frame and then using a direct linear transformation photogrammetry technique (Alem et al., 1978), the actual radial and height coordinates can be determined for a specified inflation state. This is done for several states of inflation. These coordinates, the corresponding inflating pressure and the geometrical properties of the undeformed membrane provide the data used in the identification procedure.

\section{MATHEMATICAL DESCRIPTION}

The conditions of the experiment are assumed to be appropriate for axially symmetric deformation. At each level of inflation, the membrane forms a surface of revolution. Let the polar axis of a cylindrical coordinate system coincide with the axes of symmetry of the deformed surfaces and let the origin lie in the midplane of the undeformed membrane. As shown in Fig. 1, a particle of the membrane midsurface at $(r, \theta, 0)$ in the undeformed state moves to $[\rho(r), \theta, z(r)]$ in the deformed state. In view of the axisymmetry, the principal directions of stress and stretch at each particle are known a priori. These are in the meridional 
(1) and circumferential (2) directions tangent to the membrane and also normal to the surface. The principal stretch ratios in these directions are given by, respectively,

$$
\begin{gathered}
\lambda_{1}=\frac{\mathrm{d} \zeta}{\mathrm{d} r}=\left[\left(\frac{\partial \rho}{\partial r}\right)^{2}+\left(\frac{\partial z}{\partial r}\right)^{2}\right]^{i 2}, \\
\lambda_{2}=\frac{\rho}{r}, \quad \lambda_{3}=\frac{1}{\lambda_{1} \lambda_{2}},
\end{gathered}
$$

where $d r$ is the length of a radial line element in the initial state and $\mathrm{d} \xi \xi$ is the length of the corresponding meridional element in the deformed state. The equation for $\lambda_{3}$ is determined by the incompressibility condition.

Let $\sigma_{2}(\alpha=1,2)$ denote a principal stress. Its stress resultant per unit length of a circumferential or meridional line in the membrane surface is $T_{x}=h_{0} \dot{\lambda}_{3} \sigma_{x}$. The force balance equations relative to the principal directions, transformed to the initial configuration, are

$$
\begin{gathered}
\frac{d T_{1}}{\mathrm{~d} r}+\frac{\eta\left(T_{1}-T_{2}\right)}{r \lambda_{2}}=0, \\
\kappa_{1} T_{1}+\kappa_{2} T_{2}=p,
\end{gathered}
$$

where $p$ is the inflating pressure, $\eta$ is an associated kinematic variable defined by

$$
\eta=\frac{\mathrm{d} \rho}{\mathrm{d} r}
$$

and $\kappa_{1}, \kappa_{2}$ are principal curvatures given by

$$
\begin{gathered}
\kappa_{1}=\frac{\eta \frac{\partial \lambda_{1}}{\partial r}-\lambda_{1} \frac{\partial \eta}{\partial r}}{\lambda_{1}^{2}\left[\lambda_{1}^{2}-\eta^{2}\right]^{1 / 2}} \\
\kappa_{2}=\frac{\left[\lambda_{1}^{2}-\eta^{2}\right]^{1 / 2}}{r \lambda_{1} \lambda_{2}}
\end{gathered}
$$

The invariants of the deformation tensor, in terms of the principal stretch ratios, are

$$
I_{1}=\lambda_{1}^{2}+\lambda_{2}^{2}+\lambda_{3}^{2} ; \quad I_{2}=\lambda_{1}^{-2}+\lambda_{2}^{-2}+\lambda_{3}^{-2} .
$$

Let the strain energy density function for an isotropic, incompressible, elastic solid be denoted by. $W\left(I_{1}, I_{2}\right)$. The constitutive equation is

$$
\sigma_{2}=\left(\lambda_{2}^{2}-\lambda_{3}^{2}\right)\left(W_{1}+\lambda_{B}^{2} W_{2}\right),
$$

where $W_{x}=\partial W / \partial 1_{x}$, and $\beta=2$ when $\alpha=1$, and $\beta=1$ when $\alpha=2$. The method of solving these equations for $\rho(r)$ and $z(r)$ will be presented in Section 6.

Let the radii of the material particles at the grid intersection points on the undeformed specimen be denoted by $r_{i}, i=1,2, \ldots, N$, where $N$ is the number of intersection points. The measured coordinates of a particle initially at radius $r_{i}$ will be denoted by $\left(\rho_{i}^{m}, z_{i}^{m}\right)$. The circumferential principal stretch ratio at this particle can be computed using the definition in equation (3.1). An approximation to the meridional principal stretch can be obtained from the relation

$$
\lambda_{\mathrm{t}}\left(r_{\mathrm{i}}\right)=\Delta \xi / \Delta r
$$

where $\Delta \xi$ and $\Delta r$ are the distances between material particles in the deformed and undeformed states, respectively. Finally, the invariants at this particle can be calculated from equations (3.1) and (3.6).

From the solution of the boundary value problem representing this experiment for an assumed expression for $W$, the coordinates $[\rho(r), z(r)]$ of the material coordinates at the grid intersection points $r_{i}$ are denoted by $\rho_{i}^{c}=\rho\left(r_{i}\right), z_{i}^{c}=z\left(r_{i}\right)$. The error between the calculated and measured points is defined to be

$$
E=\sum_{i=1}^{v}\left[\left(\rho_{i}^{c}-\rho_{i}^{m}\right)^{2}+\left(z_{i}^{c}-z_{i}^{m}\right)^{2}\right] .
$$

\section{DOMAIN OF DEFINITION OF $W$}

The second element of the identification procedure is a general representation for the strain energy function in terms of arbitrary parameters. Actually, in view of equation (3.7), it is more direct to construct representations for the derivatives $W_{1}\left(I_{1}, I_{2}\right)$. This requires some knowledge of the range of values taken by $I_{1}$ and $I_{2}$. Iding et al. pointed out that, owing to their definition in equation (3.6), the domain of possible values of $I_{1}$ and $I_{2}$ lies between curves determined by a pair of inequalities. Subsequently, Sawyers (1977) and Carroll (in press) independently proved that this domain is bounded by curves $A$ and $C$ shown in Fig. 2(a). Curve $A$ represents equal biaxial extension $\left(\lambda_{1}=\lambda_{2}\right)$ and curve $C$ represents uniaxial extension $\left(\lambda_{2}=\lambda_{3}=\lambda_{1}^{-1 / 2}\right)$. The straight line $B$ represents the condition $I_{1}=I_{2}$ which arises when a principal stretch ratio equals one. This is often referred to as a state of pure shear. Values for $I_{1}$ and $I_{2}$ lying between curves $B$ and $C$ arise when two principal stretch ratios are less than one. Values lying between curves $A$ and $B$ arise when two principal stretch ratios are greater than one, i.e. unequal biaxial extension. This would seem to represent the conditions appropriate to the deformation of most soft membraneous tissue.

Consider an arbitrary-candidate test specimen in a state of non-homogeneous deformation. A point in the $I_{1}-I_{2}$ plane is associated with each material particle. The points associated with the entire specimen form a domain contained between curves $A$ and $C$. As discussed by Iding et al., this domain and its variation with a change in specimen deformation is generally unknown a priori. Estimates of the extent of this domain could be made by solving the appropriate boundary value problem using assumed forms for $W$. Alternatively, an attempt could be made to measure local homogeneous deformations and compute the corresponding values of $I_{1}$ and $I_{2}$. In general, either method is tedious and leads to uncertain knowledge of the $I_{1}-I_{2}$ domain on which $W$ is to be identified. 


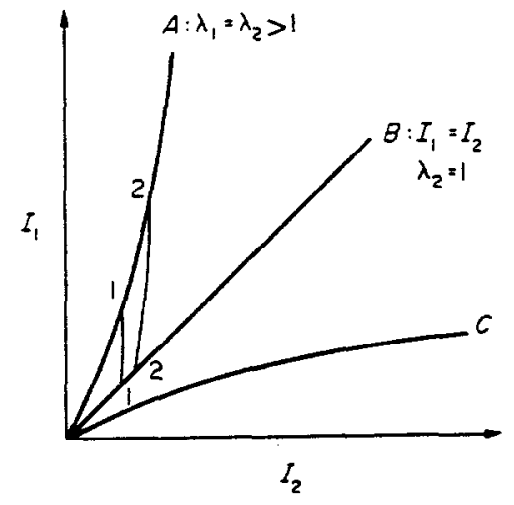

(a)
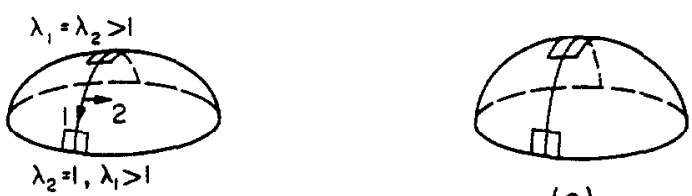

(c)

(b)

Fig. 2. Relation of inflated states to paths in the $I_{1}-I_{2}$ plane. (a) $I_{1}-I_{2}$ plane showing curves of limiting values. (b) Inflated state corresponding to path $1-1$ in the $I_{1}-I_{2}$ plane. (c) Inflated state corresponding to path 2-2.

When identification is based on the membrane inflation test, the domain of identification can be determined. Consider a fixed state of inflation. Because of the plane stress state at each material particle and the axial symmetry of deformation, $I_{1}$ and $l_{2}$ vary only in the meridional direction along the membrane midsurface. It follows that $I_{1}=g\left(I_{2}\right)$, a curve in the $I_{1}-I_{2}$ plane. Furthermore, from the a priori knowledge of the principal directions, each material particle is known to be in a state of unequal biaxial local homogeneous extension along the circumferential and meridional directions, as shown in Fig. 2(b). At the membrane center, $\lambda_{1}=\lambda_{2}>1$, an equal biaxial state. The corresponding point $\left(I_{1}, I_{2}\right)$ lies on curve $A$. At the clamped support, $\lambda_{1}>1, \lambda_{2}=1$, a state of pure shear. The corresponding point $\left(I_{1}, I_{2}\right)$ lies on curve $B$. The result is that each inflated state corresponds to an $I_{1}-I_{1}$ path connecting curves $A$ and $B$.

This path can be estimated from experimental data. The stretch ratios are essentially uniformly distributed over the central region of the membrane. Let $r_{1}=0$ and $r_{2}$ be the smallest non-zero grid radius. Then, using equation (3.1), and the notation introduced in Section 3,

$$
\lambda_{1}(0)=\lambda_{2}(0) \simeq \lambda_{2}\left(r_{2}\right)=\rho_{2}^{m} / r_{2} .
$$

Computing the invariants from equations (3.1) and (3.6) gives the point of intersection of the $I_{1}-I_{2}$ path with curve $A$. Next, using equation (3.8), $\lambda_{1}$ at the support can be estimated. Since $\lambda_{2}=1$, the value of $I_{1}=I_{2}$ can be computed and the point on curve $B$ is known. Finally, according to the discussion at the end of Section 3, $I_{1}\left(r_{i}\right), I_{2}\left(r_{i}\right)$ can be estimated at the interior grid points.

The change of the $I_{1}-I_{2}$ path with increasing levels of infiation can be discussed also. Let curve 1-1 of Fig. 2(a) represent the $I_{1}-I_{2}$ curve corresponding to the inflated state shown in Fig. 2(b). Now suppose that the membrane is further inflated as shown in Fig. 2(c). The stretch ratios $\lambda_{1}=\lambda_{2}=i$ increase at the center and $\lambda_{1}=\hat{\lambda}$ increases at the support. The corresponding invariants are

$$
\begin{array}{ll}
\text { center: } & I_{2}=2 \lambda^{2}+\lambda^{-4} ; \quad I_{2}=2 \lambda^{-2}+\lambda^{4} ; \\
\text { support: } & I_{1}=I_{2}=1+\hat{\lambda}^{2}+\hat{\lambda}^{-2} .
\end{array}
$$

The invariants corresponding to the center and support increase in value. The end points and thus the entire new $I_{1}-I_{2}$ curve move to a region of larger values. This is indicated by curve 2-2 of Fig. 2(a). According to equation (4.2), at the center $I_{1}$ increases as $\lambda^{2}$ and $I_{2}$ increases as $\lambda^{4}$, while at the support $I_{1}=I_{2}$ increases as $\hat{\lambda}^{2}$. Assuming that $\lambda_{1}$ is approximately constant over the membrane surface, the point on curve $A$ moves much more than the point on curve $B$. Consequently, the $I_{1}-I_{2}$ curve becomes approximately parallel to the $I_{2}$ axis, as shown in Fig. 2(a).

\section{REPRESENTATION FOR $w$,}

Three items are needed in order to specify a representation for $W_{2}$ : a set of basic functions of $I_{1}$ and $I_{2}$, a set of parameters and the manner in which they are to be combined. In the case of rubber elasticity, choices for these elements were deduced by inspection from the results of a large number of experiments involving homogeneous deformations. An important characteristic of each of the suggested representations is that it is defined by a single analytical expression over the entire $I_{1}-I_{2}$ plane.

Suppose $W_{2}$ is to be found for some material other than rubber using the identification method based on the membrane inflation experiment. It is useful to regard $W_{1}\left(I_{1}, I_{2}\right)$ as a surface over the domain between curves $A$ and $C$, with that between $A$ and $B$ of interest here.

Now, each inflation state corresponds to a path in the $I_{1}-I_{2}$ plane, and a section of the surface which projects onto this path (see Fig. 3). By considering a finite number of inflation states, enough sections are introduced so as to give sufficient definition to the shape of the $W_{x}$ surface. As the inflation level is increased, knowledge of this surface is extended to a larger domain.

The above suggests two approaches that can be used to construct the $W_{z}$ surface, i.e. identify $W_{z}$ from the experimental data : (1) a general representation can be constructed which holds over the entire $I_{1}-I_{2}$ domain of interest. Data from all of the inflation levels are used simultaneously to determine the parameters; (2) a separate representation is constructed for each section 


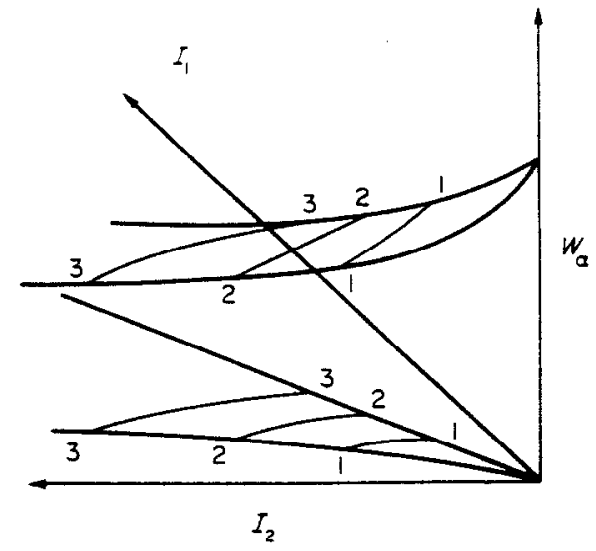

Fig. 3. Projection of $I_{1}-I_{2}$ paths onto the $W_{x}$ surface.

of the $W_{x}$ surface. The parameters are determined from each level individually.

There are several factors favoring the second approach, which is used here. First, the individual representations can be incorporated into a more general one which interpolates $W_{2}$ between the $I_{1}-I_{2}$ paths. Second, new segments of the $W_{2}$ surface can be determined as needed. Third, only a few parameters need be introduced at each step. This latter point is an advantage as far as the error minimization of element (4) is concerned.

Since results from an extensive program of homogeneous deformations will not be available to suggest the form of its dependence on $I_{1}$ and $I_{2}$, the representation for $W_{x}$ must be quite general. Following Iding et al. (1974), a finite element representation is used for this purpose. Then, along the curve $I_{1}=g\left(I_{2}\right)$,

$$
W_{1}=\sum_{i=1}^{N_{2}} A_{i}^{(x)} \phi_{i}\left(I_{1}, I_{2}\right)
$$

where $\phi_{i}$ are a set of basis functions and $A_{i}^{(2)}$ are the unknown constants. Let $\left[I_{1}^{(k)}, I_{2}^{(k)}\right], I_{1}^{(k)}=g\left[I_{2}^{(k)}\right]$, denote a set of nodal points along the $I_{1}-I_{2}$ path and at its intersections with curves $A$ and $B$. The basis functions $\phi_{i}$ are chosen as interpolation functions between the nodes. If these are linear interpolation functions, $A_{i}^{(2)}$ represent the values at the nodes. Figure 4 shows this case.

During the process of determining the constants $A_{i}^{(2)}$, a set of values is selected and the boundary value problem is solved. The error in equation (3.9) is used to modify this set, and then the boundary value problem is solved again. Each set of values and solution results in a different $I_{1}-I_{2}$ path. It is expected, however, that these paths are close to the experimentally determined one. In fact, as will be seen in the next section, all paths will share the same point on curve $A$. Thus, the method of determining the constants $A_{i}^{(\mathbf{z})}$ requires that the domain of definition of equation (5.1) be extended to a neighborhood of the experimental $I_{1}-I_{2}$ path. The simplest means of doing this consists of (a) extrapolating the basis functions at $A$ and $B$, and (b) assuming that the basis functions are independent of $I_{1}$ in some

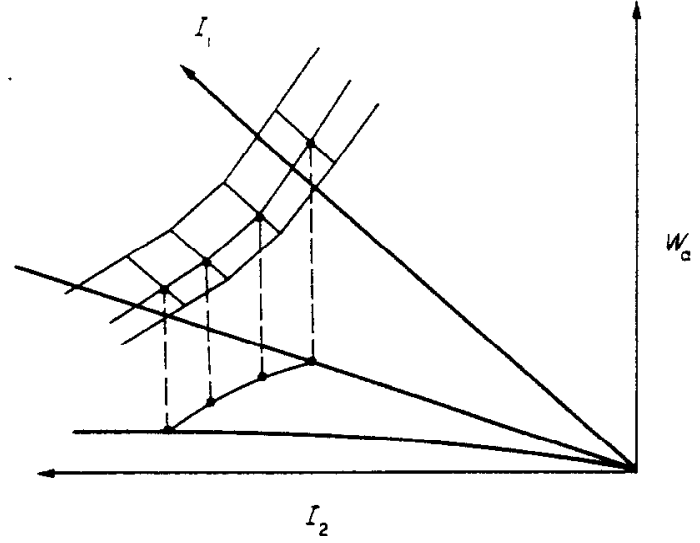

Fig. 4. Approximation of $W_{2}$ along an $I_{1}-I_{2}$ path by linear elements, extrapolation of the path.

neighborhood about the experimental $I_{1}-I_{2}$ curve. This is illustrated in Fig. 4.

It is possible to select the inflation levels so that the dependence of $W_{\mathrm{x}}$ on $I_{1}$ can be estimated. Recall from Section 4 that, as the inflation level increases, the $I_{1}-I_{2}$ paths become increasingly parallel to the $I_{2}$-axis, with the point on curve $A$ moving out faster than the point on curve $B$. As discussed in Section 3, the experimental data can be used to estimate the $A$-point quite accurately and the $B$-point adequately. Thus, successive inflation levels can be selected so that the projections of their paths along the $I_{2}$-axis overlap. Suppose nodes are selected on the two curves at the same values of $I_{2}$. Then when the constants $A_{i}^{(x)}$ have been found for the two curves, it will be possible to determine the variation of $W_{x}$ with $I_{1}$.

If $W_{2}$ can be assumed to be independent of $I_{1}$, then $W_{1}$ is independent of $I_{2}$. Identification is then greatly simplified. Suppose paths $1-1$ and 2-2 correspond to two successive inflation levels, as shown in Fig. 5, and $W_{2}$ has been identified for the former. Because a finite element representation has been used, it can be extended to the enlarged $I_{2}$ interval by adding on a new segment. The previously determined constants

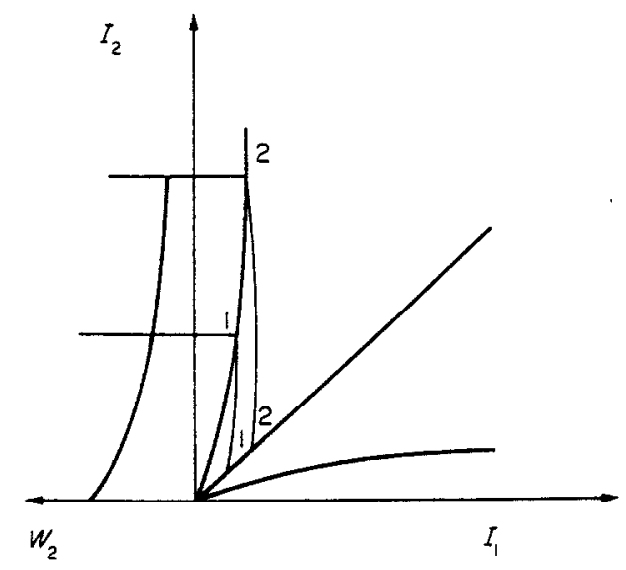

Fig. 5. Extension of the representation of $W_{2}\left(I_{2}\right)$ because of overlapping $I_{1}-I_{2}$ paths. 
can be kept fixed while the optimization procedure is used to determine the new constants. In this way, the number of constants to be determined at each step can be kept to a minimum.

\section{COMPUTING THE PARTICLE COORDINATES}

The equations presented in Section 3 can be combined to give two equations for $\rho(r)$ and $z(r)$. These form a system of coupled second order nonlinear ordinary differential equations. Most numerical procedures first reduce these to a system of first order equations. Since equations (3.2)-(3.7) already form a first order system, a great deal of effort could be saved by starting there. However, these equations can be put into a more convenient form.

First introduce the dimensionless variables $\bar{r}=r / a_{0}$, $\bar{z}=z / a_{0}, \bar{\rho}=\rho / a_{0}, \bar{\sigma}_{z}=\sigma_{z} / C_{0}, \bar{W}=W / C_{0}$ where $a_{0}$ is the membrane radius and $C_{0}$ is a convenient parameter with the dimensions of stress. By their definition in equations (3.1) and (3.4), $\lambda_{1}, \lambda_{2}$ and $\eta$ are unchanged by this non-dimensionalization. Next define the transformation

$$
\dot{\sigma}_{1}=\frac{\bar{\sigma}_{1}}{\dot{\lambda}_{1}} ; \quad \dot{\sigma}_{2}=\frac{\bar{\sigma}_{2}}{\dot{i}_{2}} ; \quad \hat{\eta}=\frac{\eta}{i_{1}} .
$$

The equilibrium equations (3.2) and (3.3), supplemented by equation (3.5), become

$$
\begin{gathered}
\frac{\mathrm{d} \dot{\sigma}_{1}}{\mathrm{~d} r}=\frac{\hat{\sigma}_{2} \bar{\eta}-\dot{\sigma}_{1}}{r}, \\
\frac{\mathrm{d} \dot{\eta}}{\mathrm{d} r}=\frac{\left(1-\dot{\eta}^{2}\right)}{r} \frac{\hat{\sigma}_{2}}{\hat{\sigma}_{1}}-P \frac{\left(1-\dot{\eta}^{2}\right)^{12} \lambda_{1} \lambda_{2}}{\dot{\sigma}_{1}},
\end{gathered}
$$

where $P=p a_{0} / h C_{0}$. Eliminating $\rho$ using $\lambda_{2}$ in equation (3.1) and $\eta$ in equation (3.4) gives a compatibility relation

$$
\frac{\mathrm{d} \lambda_{2}}{\mathrm{~d} r}=\frac{\lambda_{1} \hat{\eta}-\lambda_{2}}{r}
$$

Equations (6.2)-(6.4) define a first order system of three differential equations for $\lambda_{1}, \lambda_{2}, \eta, \hat{\sigma}_{1}, \hat{\sigma}_{2}$. The necessary additional equations are provided by combining equations (3.7) and (6.1), which can be denoted as

$$
\hat{\sigma}_{1}=\phi_{1}\left(\lambda_{1}, \lambda_{2}\right) ; \quad \hat{\sigma}_{2}=\phi_{2}\left(\lambda_{1}, \lambda_{2}\right)
$$

System $(6.2)-(6.5)$ can be reduced further. The variable $\tilde{\sigma}_{2}$ is eliminated using the second part of equation (6.5). The first part of equation (6.5) establishes a relation between $\hat{\sigma}_{1}, \lambda_{1}$ and $\lambda_{2}$. It is assumed that this can be inverted to give

$$
\lambda_{1}=\phi^{*}\left(\hat{\sigma}_{1}, \lambda_{2}\right)
$$

which can now be used to eliminate $\lambda_{1}$. The method of inversion will be discussed later. Note that the system is now reduced to three equations for $\vec{\sigma}_{1}, \hat{\eta}$ and $\lambda_{2}$,

$$
\frac{\mathrm{d} \Lambda}{\mathrm{d} r}=F(\Lambda, r, P),
$$

where $\Lambda=\left(\hat{\sigma}_{1}, \hat{\eta}_{1} \dot{\lambda}_{2}\right)^{T}$, and $F$ denotes the right hand sides of equations $(6.2)-(6.4)$.

Boundary conditions are specified at $r=0$ and $r=1$. It is shown in Green and Adkins (1960) that at $r=0, \lambda_{1}=\lambda_{2}=\eta$ and $\mathrm{d} f / \mathrm{d} r=0, f=\lambda_{1}, \lambda_{2}, \eta, \sigma_{1}, \sigma_{2}$. Then by equations (6.1) and (6.5), at $r=0$,

$$
\lambda_{2}=\lambda_{0}, \quad \hat{\eta}=1, \quad \hat{\sigma}_{1}=\phi_{1}\left(\lambda_{0}, \lambda_{0}\right)
$$

The support condition implies, by equation (3.1), that at $r=1$,

$$
\lambda_{2}=1 \text {. }
$$

Once equation (6.7) has been solved, subject to equations (6.8) and (6.9), the membrane coordinates are computed from equation (3.1). These give

$$
\begin{gathered}
\rho(r)=r \lambda_{2}(r), \\
z(r)=\int_{1}^{r} \lambda_{1}\left(1-\hat{\eta}^{2}\right)^{12} \mathrm{~d} r .
\end{gathered}
$$

There are several reasons why the problem has been formulated in this fashion. First, three dependent variables is the smallest number possible which results in a first order system to solve. An alternative system could be derived in which the three dependent variables are $\lambda_{1}, \lambda_{2}$ and $\eta$. However, this would necessitate differentiating functions $W_{x}$ in equation (3.7), which is undesirable. Secondly, $W_{2}$ can be changed without affecting the form of equations (6.2)-(6.4), the method of numerical integration and the method of obtaining the inversion (6.6). All that is needed is a change in the subroutine for computing $W_{2}$ from given values of $\lambda_{1}$ and $\lambda_{2}$.

There are several mathematical topics to be discussed. The first is the method of integrating the system of differential equations, incorporating the inversion (6.6). Suppose a fourth order Runge-Kutta integration scheme is to be used. The error in such a scheme is $O\left(\Delta r^{3}\right)$ where $\Delta r$ is the radial increment. Let the solution be known at the point $r_{i}$ and denote its values there by $\lambda_{1 i}, \lambda_{2 i}, \hat{\eta}_{i}, \hat{\sigma}_{1 i}, \hat{\sigma}_{2 i}$. The procedure for obtaining the solution at the point $r_{i+1}$ involves several applications of the following operation. The right hand sides of equations (6.2) and (6.4) are calculated using these values, multiplied by $\Delta r / 2$ and then used to increment $\hat{\sigma}_{1 i}, \hat{\eta}_{i}$ and $\lambda_{2 i}$. Denote these new values by $\hat{\sigma}_{1}^{\prime}, \hat{\eta}^{\prime}$ and $\lambda_{2}^{\prime}$. Regarded as functions of $\hat{\sigma}_{1}, \hat{\eta}$ and $\lambda_{2}$, as suggested in equation (6.7), the right hand sides are now to be evaluated at these new values. However, the actual evaluation of the right hand sides of equations (6.2)-(6.4) also requires new values for $\lambda_{1}$ and $\hat{\sigma}_{2}$. An equation for the new value of $\lambda_{1}$ is given by the first of equation (6.5):

$$
\hat{\sigma}_{1}^{\prime}=\phi_{1}\left(\lambda_{1}, \lambda_{2}^{\prime}\right)
$$

This is solved by iteration for $\lambda_{1}^{\prime}$ to give the inverse (6.6) evaluated at $\hat{\sigma}_{1}^{\prime}$ and $\lambda_{2}^{\prime}$, i.e.

$$
\dot{\lambda}_{1}=\phi^{*}\left(\hat{\sigma}_{1}^{\prime}, \dot{\lambda}_{2}^{\prime}\right)
$$

With this new value, $\hat{\sigma}_{2}^{\prime}$ is computed from equation 
(6.5). Since $\left(\dot{\lambda}_{1}^{\prime}, \ddot{\lambda}_{2}, \hat{\eta}^{\prime}, \hat{\sigma}_{1}^{\prime}, \hat{\sigma}_{2}^{\prime}\right)$ are known, the operation can be repeated.

The second topic is the method of satisfying the end conditions (6.8) and (6.9), which is as follows. The value of the stretch ratio at $r=0, \lambda_{0}$, is specified from the experimental results using the definition of $\lambda_{2}$ in equation (3.1). For each choice of the constants in the representation for $W_{x}, \hat{\sigma}_{1}$ can be evaluated. Equation (6.8) now provides initial values for the integration of equation (6.7). This can proceed as soon as a value for $P$ has been specified. Experimental data gives the radius $a_{0}$, thickness $h$ and the pressure of $p$ at each inflation level. Since the parameter $C_{0}$ is as yet arbitrary, $P$ is also arbitrary. The value for $P$ will be determined in order to satisfy equation (6.9). As a first step, a value $P_{1}$ for $P$ is assumed (or equivalently $C_{0}$ ), and equation (6.7) is integrated. Note, now, the following property of equations (6.2)-(6.4). If $r=\hat{r} / P$, the equations become independent of $P$. The solutions depend only on $\hat{r}=r P$. In particular, $\lambda_{2}=\lambda_{2}(r P)$. Suppose $\lambda_{2}=1$ at radius $r^{*} \neq 1$ for $P=P_{1}$, i.e. $\lambda_{2}\left(r^{*} P_{1}\right)=1$. Then $\lambda_{2}=1$ at radius $r=1$ at a dimensionless pressure $P_{1} r^{*}$. In actual computation, $r^{*}$ can only be estimated, since values for $\lambda_{2}$ are known only at the radial mesh points. However, a good estimate for the correct value of $P$ can be obtained by this scaling method. Improved values are then obtained by Newton-Raphson iteration.

In the numerical integration, values are provided for $W_{z} / C_{0}$ which, by equations (3.7) and (6.1), enter into the evaluation of $\dot{\sigma}_{B} / C_{0}$. For consistency, correction of the modulus in the definition of $P$ requires correction of the modulus in these evaluations. This is taken care of by regarding $W_{z} / C_{0}$ as $\left(W_{z} / r^{*}\right) /\left(C_{0} / r^{*}\right)$. Thus, if $W_{x}$ has a finite element representation (equation 5.1), the solution to the boundary-value problem corresponds to the constants $A_{i}^{(x)} / r^{*}$.

\section{DETERMINING THE CONSTANTS $A_{i}$}

According to the method outlined in the preceding section, the coordinates of the grid intersections in the deformed membrane can be regarded as functions of the constants $A_{i}^{(x)}$. By equation (3.9), the same is true of the error $E$, i.e. $E=E\left(A_{i}^{(x)}\right)$. The best choice for these constants is that which minimizes the value of $E$. The methods for calculating this optimal choice fall into two categories. The first category includes the method discussed by Iding et al. This essentially consists of deriving the system of nonlinear equations which represent the necessary conditions for a minimum of equation (3.9), i.e. $\partial E / \partial\left[A_{i}^{(x)}\right]=0$, and then solving them by an appropriate numerical procedure. Typical of the second category was the approach used by Lin and Sackman (1975) in an application of the identification method in one-dimensional visco-elasticity. An error function defined in a manner similar to $E$ was operated on directly by an optimization procedure which was developed in another context.

This latter approach is the one used in the present work. It uses a code called PRAXIS which is available in the Computing Center Library at the University of Michigan. In effect, for each choice of constants $A_{i}^{(x)}, E$ is approximated locally by a quadratic surface. Data for determining properties of this surface are obtained by evaluating $E$ at a number of neighboring choices. When sufficient data has been obtained, a new set of constants is calculated which leads to a local minimization of $E$. The process is then repeated. Each evaluation of $E$ calls, as a sub-program, the program for solving the boundary-value problem. During the evaluations, the pressure and the geometric parameters specifying the inflated state are held fixed. A set of values for the constants $A_{i}^{(2)}$ is the input data and computed coordinates $\rho_{i}^{c}, z_{i}^{c}$ are the output data. The number of evaluations of $E$ depends on the number of constants $A_{i}^{(z)}$ to be found. Thus, by using the membrane inflation test, the number of constants and hence computer costs is kept small.

The convergence criterion used in PRAXIS is met when a norm constructed from the difference of the constants determined in two successive local minimizations is less than a specified tolerance, $r_{p}$. In applications, it was found that satisfying this criterion led to longer computation time than needed. Because the experimental data is known only to a certain number of decimal places, several neighboring choices of the constants could lead to essentially the same minimum value of $E$. Successive minimizations could determine neighboring choices of those constants and not satisfy the convergence criterion of PRAXIS. Thus, a choice of constants was accepted if after an evaluation, $E$ was less than some tolerance $i_{k}$.

\section{HYPOTHETICAL EXPERIMENT}

The results of a hypothetical material identification experiment based on the membrane inflation test are discussed below. Data for this experiment were obtained by computing several inflated states using the following form of the strain energy density function proposed by Alexander (1968):

$$
W_{1}=C_{1} \mathrm{e}^{k\left(I_{1}-3\right)^{2}} ; \quad W_{2}=\frac{C_{2}}{\left(I_{2}-3\right)+C_{3}}+C_{4}
$$

$$
\text { where } \quad k=0.00015, \quad C_{1}=17.0, \quad C_{2}=19.85 \text {, }
$$
$C_{3}=0.735, C_{4}=1.0$.

The method of solving the boundary value problem, presented in Section 6, requires that a value for $\lambda_{0}$, the polar stretch ratio defined in equation (6.8), be specified. Since each inflated state corresponds to a unique value of $\lambda_{0}$, selection of the former is equivalent to selection of the latter. Inflated states and the corresponding pressures $P$ were computed for $\lambda_{0}=1.2-2.0$, with $\Delta \lambda_{0}=0.1$, as well as for $\lambda_{0}=2.5$, 3.0 and 4.0. At $j_{0}=3.0$, the equations were integrated with radial increments $\Delta r=0.02$ and $\Delta r=0.1$. Since the corresponding computed coordinates agreed to 


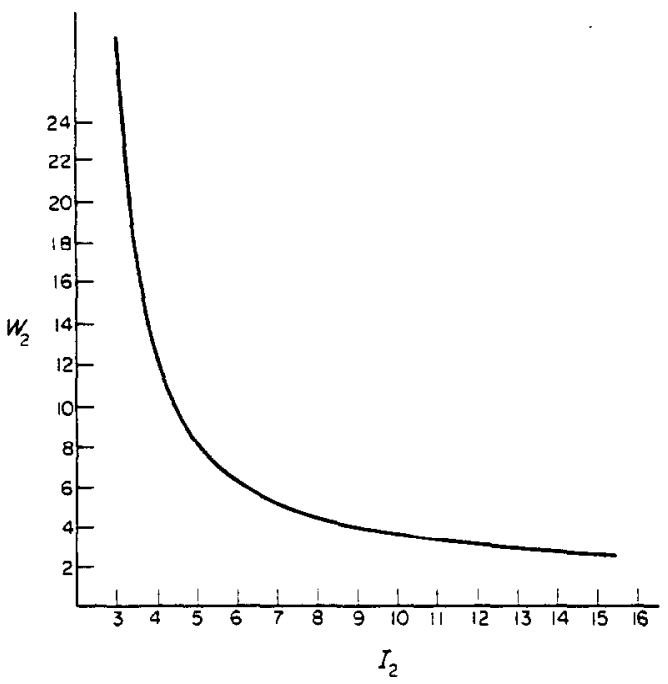

Fig. 6. Plot of $W_{2}\left(I_{2}\right)$ based on equation (8.1).

four decimal places, all further computation was carried out with $\Delta r=0.1$. The grid intersection points were chosen at $r_{i}=i a_{0} / 10$, the same points where the coordinates were computed. Finally, the dimensional pressures were computed using $h / a_{0}=0.01$ and $C_{0}=C_{1}=17.0$.

For the range of values of $\lambda_{0}$ considered, $W_{1}$ is nearly constant over the membrane. Consequently, it was decided to identify $W_{2}$ as a function of $I_{2}$. This function is shown in Fig. 6. $W_{2}$ was identified twice, first with the form shown in equation (8.1) and then with a finite element representation.

The first choice was selected as a test of how well the procedure could reproduce the constants from which data was generated. The error $E$ now becomes a function of the set $C=\left(C_{2}, C_{3}, C_{4}\right)$, the constants appearing in equation (8.1). Experimental data was taken from the inflated state corresponding to $\lambda_{0}=3.0$. For this case, $I_{2}$ varied from 3.174 at $r=1$ to 81.222 at $r=0$. Figure 6 shows that $W_{2}$ decreases rapidly on the interval $3 \leq I_{2} \leq 15$, and then more slowly to the asymptotic value $W_{2}=1$. The cross marks indicate the values of $I_{2}$ at the grid intersection points corresponding to the 'measured coordinates'. This inflation state provides data split equally between the range of rapid variation and the range of slower change.

The set $C$ was determined twice. First the 'measured coordinates', $\rho_{i}^{m}, z_{i}^{m}, i=0,1,2, \ldots 9$ were chosen to agree with the computed coordinates to 8 significant figures. The minimization process was found to be somewhat sensitive to the initial estimate $C^{0}=\left(C_{2}^{0}, \quad C_{3}^{0}, \quad C_{4}^{0}\right), \quad$ either because of the operation of the PRAXIS program or the topology of $E(\underline{C})$. For a given starting value $C^{0}$, the error $E$ would be reduced at each local minimization, and would then increase as choices for $C$ were tested for the next local minimization. This process would continue until the convergence criterion was met or the computation time limits were exceeded. When the latter occurred, computation was restarted using the best values for $C$ in the previous run. Repetition of this process let to a starting choice for the final run of $C=(20.779,0.7725,1.0324)$, which was fairly close to the desired solution. The corresponding value of $E$ was less than $10^{-3}$. The solution converged to $C=(19.851,0.7350,1.00005)$, for which $E<10^{-10}$. The maximum difference in the coordinates was $2 \times 10^{-6}$. For the second determination $\rho_{i}^{m}, z_{i}^{m}$ were equated to computed coordinates after rounding them to 3 significant figures. It was found that several choices of $C$ in some neighborhood of $(19.85,0.735$, 1.0) were equivalent in the sense that $\max \left(\left|\rho_{i}^{m}-\rho_{i}^{c}\right|\right.$, $\left.\left|z_{i}^{m}-z_{i}\right|\right)<0.001$. A typical choice is $C=(20.024$, $0.741,1.007$ ).

These results are very satisfactory. However, they are biased in that the same form of representation was used to identify $W_{2}$ as was used to generate the data. Therefore, $W_{2}$ was identified again using a finite element representation. Since $W_{2}$ depends only on $I_{2}$, the procedure discussed at the end of Section 6 was followed. That is, $W_{2}$ was identified initially in a limited $I_{2}$ domain. Then, by using higher levels of inflation, identification was extended to new intervals.

Linear elements were used initially. However, because $W_{2}$ decreases so rapidly near $I_{2}=3$, it was difficult to get satisfactory results without going to very small intervals. Consequently, it was decided to approximate $W_{2}$ by cubic polynomial elements, i.e. in the interval $x_{i} \leq I_{2} \leq x_{i+1}$ :

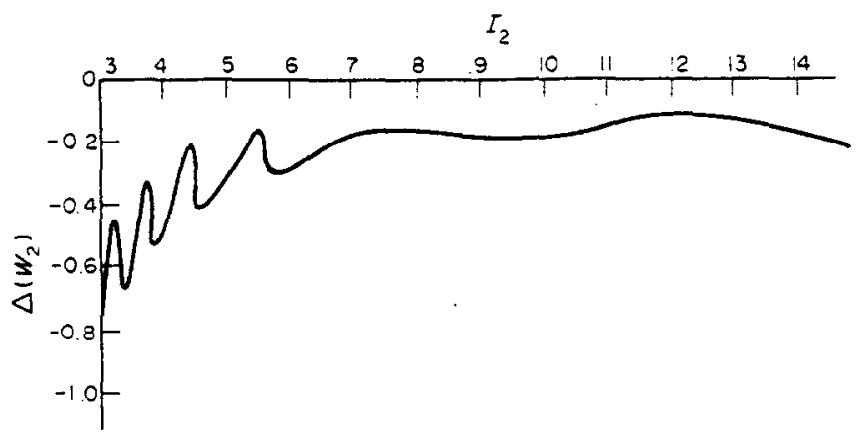

Fig. 7. Plot of $\Delta\left(W_{2}\right)=\left(W_{2}\right)_{\text {identified }}-\left(W_{2}\right)_{\text {exact }}$. 


$$
\begin{aligned}
W_{:}= & \left\{\left[A_{i}+\left(A_{i}^{\prime}\left(x_{i}-x_{i+1}\right)-2 A_{i}\right)\right.\right. \\
& \left.\times\left(I_{2}-x_{i}\right)^{\prime}\left(x_{i}-x_{i+1}\right)\right]\left(I_{2}-x_{i+1}\right)^{2} \\
& +\left[A_{i+1}+\left(A_{i+1}^{\prime}\left(x_{i+1}-x_{i}\right)-2 A_{i+1}\right)\right. \\
& \left.\left.\times\left(I_{2}-x_{i+1}\right) /\left(x_{i+1}-x_{i}\right)\right]\left(I_{2}-x_{i}\right)^{2}\right) /\left(x_{i}-x_{i+1}\right)^{2} .
\end{aligned}
$$

$A_{i}$ and $A_{i}^{\prime}$ denote the values of $W_{2}$ and $\partial W_{2}: \partial I_{2}$ at the nodes $x_{r}$. This element allows continuity of $W_{2}$ and $\partial W_{2}: \partial I_{2}$ there.

The nodal points $x_{i}$ were selected as the upper limits of the $I_{2}$ ranges covered by the solutions of the boundary value problems for $\lambda_{0}=1.2,1.3,1.4,1.5,1.7$, 1.9. These points are shown in Fig. 8. This choice appeared satisfactory to give small $I_{2}$ intervals when $W_{2}$ decreases rapidly and larger intervals when $W_{2}$ decreases more slowly.

For each inflation state, $\rho_{i}^{m}, z_{i}^{m}$ were obtained by rounding the computed coordinates to 4 significant figures. $W_{2}$ was identified on the domain $3 \leq I_{2}$ $\leq 13.586$ in 6 stages:

(1) the first element covered the interval $x_{1} \leq I_{2}$ $\leq x_{2}$. Constants $A_{1}, A_{1}^{\prime}, A_{2}, A_{2}^{\prime}$ were determined using data for $\lambda_{0}=1.2$;

(2) the second element covered the interval $x_{2} \leq I_{2}$ $\leq x_{3}$. The constants were determined using data for $j_{0}=1.3$. Initially, $A_{2}, A_{2}^{\prime}$ were kept fixed while $A_{3}, A_{3}^{\prime}$ were being determined. It was found that if for several intervals, only the constants at the right end of the interval were found, then $W_{2}$ tended to oscillate. This was eliminated by keeping $A_{1}, A_{1}^{\prime}$ fixed and determining $A_{2}, A_{2}^{\prime}$ and $A_{3}, A_{3}^{\prime}$. This procedure was followed in the remaining intervals;

(3) the third element covered the interval $x_{3} \leq I_{2}$ $\leq x_{1}$. The previously determined constants $A_{1}$, $A_{1}^{\prime}, A_{2}, A_{2}^{\prime}$ were held fixed while $A_{3}, A_{3}^{\prime}$ and $A_{4}$, $A_{4}^{\prime}$ were to be found using data for $x_{0}=1.4$. The change in $A_{3}, A_{3}^{\prime}$ would affect the fit of the element in the interval $x_{2} \leq I_{2} \leq x_{3}$. The data for $\lambda_{0}=1.4$ provided only two points in this interval, as can be seen from Fig. 8, which is not a sufficient number to ensure a proper fit of the element. To overcome this difficulty, it was decided to incorporate the data for $\lambda_{0}=1.3$.

To accomplish this, the error $E$ was generalized. Let $E^{(x)}$ denote the square error defined in equation (3.9) for the ath inflated state. Then the generalized error is

$$
E=\sum_{x=1}^{y} E^{(x)}
$$

where $N$ is the number of states to be considered. The program was modified in two ways: (a) to accommodate the increased amount of input data, i.e. measured coordinates, polar stretch ratios and pressures for each inflation level; (b) for each evaluation of $E$, the boundary value

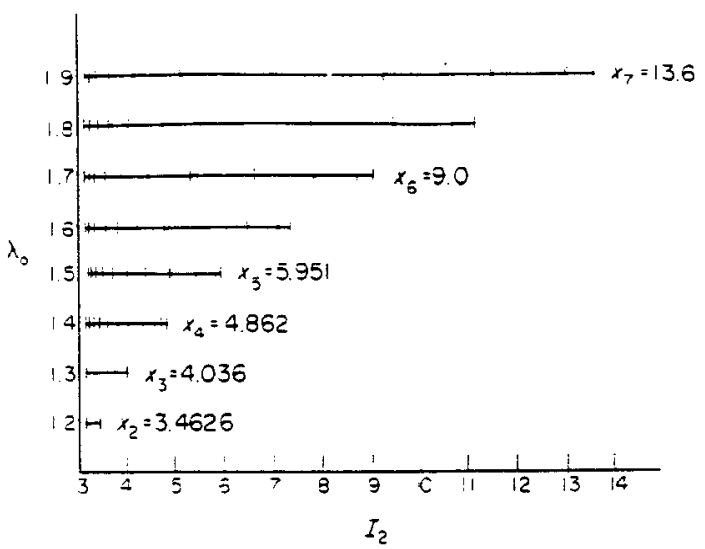

Fig. 8. Range of $I_{2}$ values for various inflated states, and choices for nodal points $x_{i}$. $i_{0}$ denotes the stretch ratio at $r=0$. The vertical marks denote $I_{2}$ values at the grid radii.

problem would have to be solved for the $N$ inflated states;

(4) the fourth element covered the interval $x_{4} \leq I_{2}$ $\leq x_{5}$. Constants $A_{4}, A_{4}^{\prime}, A_{5}, A_{5}^{\prime}$ were determined using data for $\lambda_{0}=1.5$ and $\lambda_{0}=1.4$. The latter set provided points in the interval $x_{3} \leq I_{2} \leq x_{4}$;

(5) the previous results indicated that $W_{2}$ is decreasing less rapidly. The next element covered a longer interval, $x_{3} \leq I_{2} \leq x_{6}$. Data for $\lambda_{0}=1.6$ and $i_{0}=1.7$ were used;

(6) the last element considered covered $x_{6} \leq I_{2}$ $\leq x_{7}$. The constants were determined from data for $\lambda_{0}=1.8$ and $\lambda_{0}=1.9$.

Values for the constants were accepted if either (a) the PRAXIS program tolerance $i_{p}<10^{-2}$ or (b) the error tolerance $\varepsilon_{F}<10^{-8}$. Stages $(1)-(4)$ and (6) converged by (b) and stage (5) by (a). For the first element, the initial guess for the constants was determined by experience with the linear elements. For the later elements, the initial guess was obtained by linear extrapolation from the results at the right end of the previous element. About 150 evaluations of $E$ were required at each stage in order to obtain a satisfactory choice for the constants.

For the identified $W_{2}$, the fit of the computed profiles to the measured profiles was very good, with a maximum error of about $0.1 \%$. The identified $W_{2}$ also compared very well with the exact form given by equation (8.1). Table 1 compares the nodal constants of the identified $W_{2}$ with those calculated from equation (8.1). Figure 7 shows the difference between the identified $W_{2}$ and that given by equation (8.1) on the entire range of identification.

As was mentioned under stage (2), whenever a new element was added, the constants at both ends of the interval were determined by the minimization. This meant that the constants at nodes $x_{2}-x_{6}$ were determined twice. Table 2 shows the changes produced by the second determination. As would be expected, the biggest change was in the slopes. 
Table 1

\begin{tabular}{ccccc}
\hline Nodal point & $\left(W_{2}\right)_{E}$ & $\Delta W_{2}$ & $\left(\partial W_{2} / c I_{2}\right)_{E}$ & $\Delta\left(\partial W_{2} / \partial I_{2}\right)$ \\
\hline 3.0 & 28.007 & -0.752 & -36.744 & 1.559 \\
3.4626 & 17.575 & -0.611 & -13.840 & 1.391 \\
4.036 & 12.208 & -0.45 & -6.329 & 0.7326 \\
4.8620 & 8.643 & -0.347 & -2.9432 & 0.3641 \\
5.951 & 6.3852 & -0.2935 & -1.4610 & 0.1237 \\
9.0 & 3.9473 & -0.1985 & -0.4376 & -0.0079 \\
13.6 & 2.7512 & -0.1542 & -0.1545 & -0.0305 \\
\hline()$_{E}$ means computed from equation $(8.1),\left(\right.$ ) means identified value. $\Delta(\quad)=()_{t}$ \\
-()$_{E}$.
\end{tabular}

Table 2

\begin{tabular}{cccccc}
\hline & \multicolumn{2}{c}{ First determination } & & \multicolumn{2}{c}{ Second determination } \\
\cline { 2 - 3 } \cline { 5 - 6 } Nodal point & $\left(W_{2}\right)_{l}$ & $\left(\partial W_{2} / \partial I_{2}\right)_{l}$ & & $\left(W_{2}\right)_{l}$ & $\left(\partial W_{2} / \partial I_{2}\right)_{l}$ \\
\hline 3.0 & 28.0 & -22.0 & & - & - \\
3.4626 & 17.0 & -22.0 & & 17.0 & -11.0 \\
4.036 & 12.5 & -6.0 & & 12.0 & -5.0 \\
4.862 & 8.0 & -3.5 & & 8.3 & -2.6 \\
5.951 & 6.3 & -1.3 & & 6.0 & -1.4 \\
9.0 & 3.8 & -0.6 & & 3.7 & -0.44 \\
13.6 & 2.4 & -0.2 & & - & - \\
\hline
\end{tabular}

The use of the finite element representation in the integration of equation (6.7) requires discussion of a final computation issue. In computing the solution at $r_{j+1}$ by the Runge-Kutta method, it is assumed that the functions have a continuous fourth derivative. Suppose that $I_{2}\left(r_{j}\right)$ is in the domain of one basis function and $I_{2}\left(r_{j+1}\right)$ is in the domain of the next, so that it becomes necessary to change from one to the other within the interval. Since $W_{x}$ will not have this required continuity at the nodal points of its representation, the integration scheme may not give the correct result. This difficulty is overcome by following the suggestion of Iding et al. The same basis function is used for all calculations in the interval between $r_{j}$ and $r_{j+1}$. If this interval is sufficiently small and the variation of the solution sufficiently slow, the error induced by this assumption is likely to be small. The satisfactory results described here suggest that this seems to be the case.

Acknowledgement - The authors gratefully acknowledge support for this work under NSF Grant No. 75-04267.

\section{REFERENCES}

Adkins, J. E. and Rivlin, R. S. (1952) Large elastic deformations of isotropic materials, IX. The deformation of thin shells. Phil. Trans. R. Soc. (A) 244, 505-531.

Alem, N. M., Melvin, J. W. and Holstein, G. L. (1978) Biomechanics applications of direct linear transformation in close range photogrammetry. Proceedings of the Sixth New England Bioengineering Conference. Pergamon Press, New York.

Alexander, H. (1968) A constitutive equation for rubber-like materials. Int. J. Engng. Sci. 6, 549-563.

Carroll, M. M., J. Elasticity. In press.

Green, A. E. and Adkins, J. E. (1960) Large Elastic Deformations and Non-Linear Continuum Mechanics. Clarendon Press, Oxford.

Hart-Smith, L. J. and Crisp, J. D. C. (1967) Large elastic deformations of thin rubber membranes. Int. J. Engng. Sci. $5,1-24$.

Iding, R. H., Pister, K. S. and Taylor, R. L. (1974) Identification of non-linear elastic solids by a finite element method. Computer Methods App. Mech. Engng. 4, 121-142.

Klingbeil, W. W. and Shield, R. T. (1964) Some numerical investigations on empirical strain energy functions in the large axi-symmetric extensions of rubber membranes. $Z$. angew. Math. Phys. 15, 608-629.

Lin, E. I.-Ho and Sackman, J. L. (1975) Identification of the dynamic properties of nonlinear viscoelastic materials and the associated wave propagation problem. Int. J. Solids Structures 11, 1145-1159.

Melvin, J. W., Mohan, D. and Wineman, A. S. (1975) Mechanical characteristics of soft tissues at high strain rates. Proc. 1975 Biomech. Symp. ASME. 75-78.

Miller, L. E., Lavery, J. P. and Donnelly, T. A. (1979) Determination of the elastic parameters for human fetal membranes. J. Rheol. 23, 57-78.

Sawyers, K. N. (1977) On the possible values of the strain invariants for isochoric deformations. J. Elasticity 7 , 99-102.

Treloar, L. R. G. (1944) Strains in an inflated rubber sheet, and the mechanisms of bursting. Inst. Rubber Ind. Trans. 19, 201. 